Denise Douzant-Rosenfeld, Bogumiła Lisocka-Jaegermann, Jerzy Makowski, Maria Skoczek, Jean-Christian Tulet

\title{
LES CHANGEMENTS DANS LA MISE EN VALEUR TOURISTIQUE DE LA RÉGION DE MAZURIE. EXEMPLE DES GMINAS DE MIKOŁAJKI ET PIECKI ${ }^{1}$
}

L'agro-tourisme en Pologne, bien que de fraîche date, est de plus en plus présent. Son rôle dans le développement des espaces ruraux a été reconnu en 1994 dans deux programmes gouvernementaux concernant les principes de la politique agricole et ceux de la gestion des activités touristiques. Dans les années quatre-vingt-dix, plusieurs associations agro-touristiques régionales ont été créées, bénéficiant de l'aide prévue dans les programmes d'intégration à l'Union Européenne. Une fédération ("Exploitations d'hospitalité"), qui les a regroupées, a introduit un système commun de standarisation et de catégorisation des services fournis par les agriculteurs. Elle comptait plus de 1000 exploitations, c'est-à-dire $25 \%$ env. des exploitations accueillant des touristes (Polska. Atlas agroturystyczny, 1999). La filiale polonaise de l'ECEAT (Centre européen pour l'agriculture écologique et le tourisme) a été créée en 1993. Dans les administrations agricoles, une formation des agriculteurs en vue de la promotion de l'agro-tourisme a été aussi entreprise. Il faut enfin noter l'activité des autorités locales, dans le cadre de l'autonomie territoriale: amélioration des routes, des canalisations et du traitement des eaux, promotion et aide aux exploitations agro-touristiques.

Ces dernières années, la production agricole en Pologne étant de moins en moins rentable, les services touristiques apparaissent donc comme une importante source de revenu. Cela concerne surtout les petites exploitations, localisées dans les régions touristiquement intéressantes et ayant déjà une certaine expérience de l'accueil de touristes. Ce sont surtout les "néo-ruraux"

\footnotetext{
${ }^{1}$ Ce rapport fait partie d'une série d'études réalisées dans le cadre d'un projet de recherche "Le tourisme comme facteur du développement local et régional en Europe et en Amérique Latine", entrepris en collaboration avec des géographes argentins (de l'Université de Neuquén) et français (des Universités de Toulouse et de Paris-Nanterre). Les données, recueillies en juin 1999, dans les deux gminas - ensembles de communes faisant partie d'une division administrative de base - font suite aux enquêtes menées en juin 1991 par l'équipe LanguedocMazowsze qui, depuis 1981, réunit des géographes et des économistes de l'Université de Montpellier III, des Universités de Varsovie et de Eódź, ainsi que de l'Académie Polonaise des Sciences.
} 
qui se lancent dans ce type d'activité. Jeunes, nés en ville, souvent diplômés d'écoles d'agriculture, ayant délibérément choisi la campagne comme lieu de travail et de résidence. Il est en effet significatif que la majorité des exploitations faisant partie de l'association "Exploitations d'hospitalité" se trouvent dans la région de Bieszczady, dans le Sud-Est, et justement dans la région de Mazurie - c'est-à-dire là où la grande partie de la population rurale n'est pas originaire de ces régions.

Il y a encore, à la campagne, une catégorie particulière de personnes qui misent sur le tourisme: il s'agit de ceux qui investissent leur argent gagné à l'étranger dans la construction immobilière. Cela concerne les régions de montagne situées dans le Sud de la Pologne - et la région de Mazurie, où les migrations de travail, surtout vers l'Allemagne, sont importantes et le transfert d'argent en provenance des familles établies à l'étranger est fréquent.

Il est certain que grâce au développement de l'agro-tourisme l'état de l'infrastructure et de l'habitat rural s'est très souvent amélioré. Parfois les habitants sont devenus plus dynamiques, p. ex. dans l'organisation de manifestations folkloriques. Il est à souligner que leur sensibilité vis-à-vis du milieu naturel est de plus en plus grande et que leur identité régionale apparaît (ou réapparaît). Le rôle de l'agro-tourisme dans la création d'emplois et dans le ralentissement de l'exode rural (vers les villes, à l'étranger) est par contre discutable car, comme cela a été dit, cette nouvelle activité concerne surtout des personnes extérieures aux espaces ruraux. Elle est aussi limitée aux régions les plus intéressantes du point de vue de l'afflux de touristes.

La région de Mazurie constitue justement une de ces régions - et sa partie méridionale, où nous avons enquêté, est la plus connue et le plus souvent visitée par les touristes. Son relief, post-glaciaire, vigoureux et très varié, est une valeur indiscutable. Ses lacs, entourés de forêts, offrent un paysage pittoresque. Un ensemble de 24 lacs, couvrant $300 \mathrm{~km}^{2}$, reliés entre eux par un système des canaux, constitue une attraction particulière. Une quinzaine de lacs de plus de $1 \mathrm{~km}^{2}$ et quelques dizaines de lacs plus petits, mais dépassant 1 ha, complète cette richesse aquatique. Dans l'ensemble, les lacs occupent $350 \mathrm{~km}^{2}$ env. (20\% de la superficie de la région). Ils permettent de pratiquer la voile pendant l'été, le bateau sur patins pendant l'hiver. Ajoutons à cela les canaux et les rivières navigables. Les forêts constituent une autre richesse, surtout dans le Sud de la région. Elle est donc aussi vouée à d'autres formes de tourisme: randonnées pédestres, excursions en vélo, safaris photographiques. Les terrains les plus intéressants du point de vue du milieu naturel sont protégés, comme ceux du Parc naturel de Mazurie $\left(521,6 \mathrm{~km}^{2}\right)$, instauré en 1977.

Les valeurs d'ordre culturel sont beaucoup moins importantes. Les touristes visitent surtout les édifices d'architecture médiévale et les musées régionaux, localisés en principe dans les villes. À cause de l'histoire compliquée et tourmentée de la région, plusieurs vestiges du patrimoine culturel restent oubliés, certains dépérissent. Les palais et les manoirs appartenant jusqu'en 1945 aux grands propriétaires terriens prussiens sont 
peu connus - ainsi que, dans la campagne, les constructions traditionnelles en bois, dont les ensembles entiers villageois. Peu d'attractions sont offertes aux touristes. La plupart de manifestations proposées pendant l'été concerne les concerts de jazz où de la chanson contemporaine. Les tentatives d'organisation de manifestations se référant à la culture locale sont rares.

La région de Mazurie est une des régions les mieux mises en valeur du point de vue de tourisme. Elle réunit $12 \%$ de l'offre globale d'hébergement du pays (Lijewski, Mikułowski, Wyrzykowski, 1998). Avant 1989 elle était concentrée dans des ensembles appartenant aux entreprises et institutions ayant leur siège social surtout à Varsovie. Une bonne partie de lits se trouvaient dans les bases nautiques, dans les campings et dans les terrains de bivouac, administrés habituellement par une sorte d'association monopoliste de tourisme. Plus de $80 \%$ de cette offre d'hébergement ne fonctionnait que pendant l'été.

Depuis le changement de régime, et à des rythmes différents, toutes les formes d'hébergement et d'activités touristiques ont été développées, autour du concept de tourisme vert respectueux de l'environnement et devant permettre un "développement durable": grands hôtels type riviera sur quelques sites choisis, hôtels et pensions, gîtes ruraux dispersés, campings, etc. et bien sûr transformation du tourisme social des maisons de vacances. L'équipement des régions rurales en téléphones et routes permet un meilleur accès des citadins aux zones les plus reculées. La Mazurie est à 3 heures de route de Varsovie. On peut y venir en fin de semaine et en vacances toute l'année. Maisons et terrains agricoles sont d'autant plus disponibles que l'agriculture, l'élevage laitier et les industries qui y sont liées subissent une crise profonde.

Dans les gminas de Mikołajki et de Piecki l'offre d'hébergement est relativement importante; dans les années quatre-vingt-dix quelques initiatives locales ont aussi eu lieu, en vue de la promotion du tourisme. Les enquêtes, menées dans ces gminas et dans quelques endroits dans les gminas voisines (celle de Mragowo et de Ruciane-Nida) s'adressaient:

- aux autorités municipales et quelques responsables d'associations;

- aux propriétaires de fermes agro-touristiques, offrant des chambres d'hôtes;

- aux propriétaires des hôtels et pensions de petite taille, où la fonction agricole a quasiment disparu;

- aux employés ou les propriétaires des attractions et hébergements touristiques, s'appuyant sur l'environnement et l'histoire locale: parc naturel (Mazurski Park Krajobrazowy), parc animalier (Park Dzikich Zwierzqt à Kadzidłowo), bases nautiques, complexe Galindia - Mazurski Eden, musée Chata Mazurska à Sądry.

À la fin des années quatre-vingt-dix, la situation dans les deux gminas était la suivante:

La gmina de Piecki:

Cet ensemble rural ( $315 \mathrm{~km}^{2}, 8500$ habitants dont 3300 dans le bourg) 
occupe une situation prometteuse sur la route des lacs. On y vivait traditionnellement de l'agriculture, aujourd'hui en crise, et d'activités de services touristiques peu intégrées au développement local et fournissant très peu d'emplois: 19 établissements de vacances appartenant aux entreprises ou à l'État, quelques campings ou pensions de taille moyenne, une base nautique, mais pas d'hôtel à capacité suffisante pouvant attirer les clientèles citadine et allemande: au total près de 2500 lits, pour des séjours de deux semaines en moyenne en haute saison (juillet-août) et 10000 touristes l'an. $30 \%$ de chômeurs sont officiellement recensés dont beaucoup de jeunes désoeuvrés et peu d'activités nouvelles: quelques commerces, une fabrique de meubles fondée par un Allemand, et les lents débuts du tourisme à la ferme.

\section{La gmina de Mikołajki:}

En dépit de sa taille encore relativement modeste ( 4000 habitants permanents), le bourg de Mikołajki tend progressivement à se transformer en un pôle de développement touristique de première importance. Ce développement touristique doit être mis en relation avec un site exceptionnel, à la confluence de plusieurs lacs, le bourg lui-même se localisant sur une presqu'île, au débouché d'un pont enjambant un bras de ces multiples lacs. La situation de Mikołajki est également à prendre en compte, comme point de passage obligé de la circulation lacustre.

Les installations d'hébergement situées dans la ville elle-même ne sont pas encore exceptionnelles: 4 hôtels, trois centres de repos, un camping, sept pensions, une trentaine ou une quarantaine de possibilités d'hébergement dans des maisons particulières. Tout cela ne signifierait pas grand chose sans la présence, à l'entrée du bourg, du plus grand hôtel de la région (Hotel Gołębiewski): 1200 chambres pouvant accueillir au total 1500 personnes. Cet hôtel est ouvert toute l'année. Il fonctionne également comme un centre de conférence d'une capacité d'un millier de personnes.

C'est la construction de cet hôtel qui a suscité le développement du tourisme de "masse" dans la région. On enregistre pendant la grande période touristique jusqu'à 10000 personnes/jour. C'est lui qui a également provoqué le développement de capacités d'hébergement supplémentaires. Actuellement ces capacités atteindraient, tout compris, 3000 chambres. Il favorise également l'allongement de la saison touristique qui serait actuellement de six mois. Cet équipement important favorise bien évidemment les activités commerciales ainsi que, selon nos interlocuteurs, la vente des produits régionaux. Il existe des accords de coopération entre l'hôtel et d'autres établissements de la ville, voire de la région.

La réussite de l'implantation de ce premier très grand établissement provoque à l'évidence un effet d'entraînement très puissant. De très grands projets ont vu le jour. Une société de Varsovie compte prochainement démarrer un centre récréatif de type "marina", un golf de 27 trous et avec des capacités d'hébergement supérieures au grand hôtel actuel.

D'après les études effectuées en 1991, la fonction touristique n'avait 
d'importance que dans neuf sur trente-un villages situés autour de Mikołajki; les autorités locales prévoyaient que dans quatre villages seulement l'infrastructure touristique se développerait (Rapport..., 1991). Huit ans après nous avons constaté l'accroissement de diverses formes d'hébergement dans plusieures localités. La présence des exploitations agro-touristiques est de plus en plus visible: il y en avait 12 dans la gmina de Mikołajki et 15 dans la gmina de Piecki. La concurrence entre elles conduit à diversifier les services qu'elles fournissent et à améliorer leur qualité. Depuis quelques années certaines fermes agro-touristiques se spécialisent dans l'élevage de chevaux et dans l'organisation des "vacances en selle". Étant donné la crise de l'agriculture plusieurs fermiers ont misé sur le tourisme. Il arrive aussi que les exploitations soient achetées par des personnes arrivant de l'extérieur, pour organiser les chambres d'hôtes où pour construire un hôtel. La plupart d'eux ne possédaient pas avant d'exploitations agricoles, mais certains sont diplômés d'écoles d'agriculture.

En 1994 les exploitations touristiques de la gmina de Piecki disposaient de 500 lits, en 1999 - de 2000 lits env.; leurs propriétaires s'associent dans des organisations professionnelles. La gmina de Mikołajki comptait 7 hôtels, outre les lits dans les exploitations agro-touristiques; dans certaines, des activités peu habituelles se sont développées (élevage de cerfs, élevage de poissons, organisation des séjours de sculpture artistique). L'offre d'hébergement est très variée: depuis un complexe touristique de luxe à Galindia-Mazurski Eden, fréquenté surtout par les employés des entreprises étrangères, au bord du lac, avec 20 ha de terrains agricoles et forestiers, 100 lits dans 43 chambres et 100 emplacements dans le camping - jusqu' aux chambres d'hôtes, bien modestes (2-4 pièces dans la maison du propriétaire).

\section{Voici quelques exemples, les plus typiques:}

1. Ferme d'agriculture écologique à Użranki (gmina de Mragowo): un couple jeune, de formation agronomique, originaire de Varsovie, ayant tra-vaillé comme techniciens dans différentes fermes d'État, avant de s'installer à leur compte au début des années 90 , avec leurs parents. Ils exploitent 50 ha (terres propres et en location) pour nourrir les animaux: quelques vaches et porcs, et surtout un troupeau d'une centaine de chèvres fournissant 250 litres de lait/jour en moyenne. Sept personnes travaillent: trois pour les animaux, trois pour la laiterie dont un ou deux volontaires, car la ferme, modernisée, reçoit des stagiaires en agronomie. Une laiterie moderne et sanitairement contrôlée a été installée. Ils militent pour convaincre des éleveurs voisins de se lancer dans le même créneau afin d'arriver à 1000 litres/jour et rentabiliser l'installation. Leur meilleur argument est le prix du lait, acheté trois fois plus cher que la laiterie de la zone. Les fromages de chèvre sont vendus par eux-mêmes une fois par semaine dans différents points de vente à Varsovie, et sur place. L'agro-tourisme a été ajouté en 1994, pour diversifier les activités: le camping à la ferme et deux chambres en location (6 lits) dans la maison traditionnelle, avec pension complète et table d'hôte permet d'accueillir en été des familles, avec ou sans enfants, des personnes variées, souvent étrangères (hollandais, allemands, danois...), à travers le réseau ECEAT auquel ils appartiennent. Le tourisme fournit environ $15 \%$ des revenus.

2. Ferme agro-touristique à Faszcze (gmina de Mikołajki). La ferme de 20 ha plus 10 ha en location, achetée en 1976, est encore bien fonctionnelle en dépit des difficultés, et produit pour l'élevage: quelques vaches, mais surtout une porcherie de 100 têtes. Un chalet en bois a été construit dans le jardin, avec deux chambres d'hôtes, salle de bain, cuisine. La fermière 
et ses deux enfants, déjà dans la vie active, reçoivent surtout des familles en été. Le tourisme permet le maintien de l'activité agricole ainsi que les salaires des enfants qui vivent encore à la maison.

3. Ferme agro-touristique à Nowy Most (gmina de Piecki), avec camping à la ferme équipé et un nouveau bâtiment de 5 chambres avec salle de bain (12 lits). Leur ferme de 10 ha (aujourd'hui 17 ha exploités en prairies, plus des terres louées), héritée de leur père, est toujours active. L'élevage laitier permettait de vivre jusqu'en 1990. Aujourd'hui il est si peu rentable qu'ils pensent à ne garder que deux vaches pour la consommation touristique, et l'élevage des veaux plus rémunérateur. La basse-cour, le potager fournissent les produits fermiers suffisants pour leurs hôtes. C'est aujourd'hui l'activité touristique qui finance la ferme, dirigée avec l'aide des deux enfants, étu-diants en hôtellerie et cuisine.

4. Ferme transformée en auberge rurale avec musée Chata Mazurska à Sądry (gmina de Mrągowo). Le créneau exploité est le tourisme "nostalgique" allemand. Les propriétaires du lieu sont des polono-allemands locaux qui n'ont pas émigré. La ferme familiale de 30 ha a été exploitée jusqu'au début des années 90 , puis progressivement abandonnée au profit de l'accueil en chambres d'hôtes, puis de la construction d'une auberge confortable de 16 chambres, avec pension, bar et terrasse, grand jardin d'agrément et espace couvert pour les réceptions, que la propriétaire exploite avec son fils (qui a fait l'école hôtelière) et trois employés. La maison rurale prussienne a été convertie en musée regroupant mobilier et ustensiles traditionnels, mentionné comme attraction locale dans les guides et surtout chez les tours-opérateurs allemands qui organisent les voyages en autobus dans la région, en particulier de gens du $3^{e}$ âge.

5. La pension Kos à Zgon (gmina de Piecki), sur la route touristique, avec terrasse et jardin, bar-restaurant, hôtel ( 8 chambres, 18 lits), camping équipé pour 10 emplacements qui peut recevoir des groupes organisés (en demi-pension souvent) ou des individuels (couples, familles) polonais et également étrangers. En accord avec une agence de Varsovie, ils servent en particulier de point d'accueil pour des groupes de sportifs allemands pratiquant le kayak. L'activité a commencé en 1992 par l'achat et l'équipement d'une vielle maison sur la route. Le potager, le verger, la basse-cour familiaux fournissent des produits appréciés par la clientèle.

6. Le centre nautique de Babięta, en bordure de la rivière de la route des lacs, de la forêt et accessible aussi par la route (pour itinérants et séjours) offre de mai à octobre, restaurant rénové, emplacements de camping limités volontairement à 50 personnes, et chalets-bungalows bien entretenus (24 en tout). Si les pratiquants de kayaks continuent à fournir $50 \%$ de la clientèle, la clientèle restante vient pour les activités de nature, la pratique du cheval (à proximité) pour des séjours de plusieurs semaines. La YMCA y organise son congrès tous les deux ans. La gérante et sa famille, originaires de la localité, emploient 10 employés saisonniers.

En somme, voici quelques conclusions concernant le développement de l'agro-tourisme dans les deux gminas étudiées:

- les fonds nécessaires à la mise en route des exploitations agrotouristiques proviennent surtout des migrations de travail en Allemagne, des activités hors agriculture, de la vente d'une partie des terres disponibles; les crédits préférentiels, obtenus en 1994-97 et actuellement péniblement remboursés, ont été très peu utilisés;

- les propriétaires des exploitations agro-touristiques sont plus instruits que les agriculteurs; certains appartiennent à la catégorie des "néoruraux", venant des villes, dont Varsovie;

- dans les exploitations agro-touristiques, en dehors des "exploitations écologiques", l'agriculture ne joue qu'un rôle modeste; parfois, elle est complètement abandonnée, on construit des hôtels et on s'efforce de créer les conditions permettant d'accueillir les touristes pendant toute l'année;

- les propriétaires des exploitations agro-touristiques voient l'avenir de 
leurs enfants dans le tourisme; en effet, ces enfants sont habituellement envoyés dans des écoles de tourisme. En l'absence d'une alternative agricole réellement opérationnelle (sols pas toujours de bonne qualité, perspectives de reconversion des structures agraires pas encore très claires), l'option du développement touristique adoptée par un certain nombre de familles locales, constitue de toute évidence une option pertinente. Elle valorise un milieu relativement attractif et des moyens disponibles.

La disponibilité de fermes classiques à prix abordable après le dernier départ des polono-allemands dans les années soixante-dix a permis à des "néo-ruraux" venus de la ville et à des paysans venus de régions rurales denses du Sud et du centre du pays de s'installer en Mazurie et d'y promouvoir des activités novatrices: agriculture écologique, tourisme vert, activités sportives et culturelles.

Les innovateurs ont mis l'accent sur la volonté et la nécessité d'étendre la saison touristique au-delà de la haute saison traditionnelle d'été (vacances scolaires), en promouvant les formules de week-end toute l'année pour les gens des grandes villes comme Varsovie, les vacances de Noël et le Jour de l'An, les vacances d'hiver (neige, lacs gelés), les séjours de touristes internationaux du $3^{\text {e }}$ âge... Il s'agit là d'une obligation impérative, les infrastructures de ce genre de tourisme ne pouvant nulle part être amorties sur la seule période estivale. En France, pour ces mêmes raisons, une bonne part des investissements à usage collectif (bases nautiques, équipements récréatifs ...) sont pris en charge par l'État ou par les collectivités locales, les associations ou les particuliers recevant des subventions diverses afin d'atteindre le seuil minimum de rentabilité. Il ne nous est pas apparu qu'une telle politique soit à l'ordre du jour en Pologne.

Les enquêtes nous paraissent révéler l'absence d'une politique régionale de développement, dans laquelle le tourisme pourrait prendre sa place, à côté d'autres activités. Pour l'instant, tout semble être laissé à l'initiative individuelle, ce qui risque de faire progressivement désordre et d'entraîner rapidement divers blocages. L'option "tourisme vert "peut être un choix, facilité en cela par les qualités du milieu et le tourisme "nostalgique allemand". Mais il risque d'être incompatible avec l'absence d'un plan d'aménagement général de la région. Celui-ci supposerait, entre autres, un zonage préservant des espaces protégés, inconstructibles, et l'application de règles pour la mise en place de nouveaux équipements, lotissements ou constructions, préservant l'environnement (stations d'épuration, etc.). Une action des collectivités ou de l'État serait également la bienvenue afin de faciliter la reconversion des fermes d'État.

En tout état de cause, la seule option touristique pour le développement régional nous paraît quelque peu dangereuse. Les activités agricoles, qui semblent aujourd'hui largement délaissées, sauf à servir de caution pour continuer à bénéficier d'exemptions fiscales, mériteraient probablement d'être mieux prises en compte, y compris sous la forme du développement de produits "alternatifs", comme nous en avons déjà vu divers exemples. 
L'entretien des paysages, préalable incontournable de ces formes de tourisme, s'effectue d'abord par le maintien d'une activité agro-pastorale.

Cette région constitue un exemple très intéressant de la diversité des problèmes de transition que doivent affronter la plupart des campagnes polonaises. Il s'agit, en plus, d'une contrée particulière, dominée jadis par les grandes fermes d'État, actuellement ruinées - et où le taux de chômage est très élevé (24\%, alors que la moyenne nationale est de $13 \%$ ). Les campagnes doivent inventer de nouvelles stratégies, sous peine de plonger durablement dans une crise profonde et déstabilisatrice pour tout le tissu social. L'observation du sens pris par l'évolution actuelle de ces stratégies nouvelles de production nous semble donc constituer un objet d'étude fondamental, non seulement pour l'analyse des dynamiques sociales en cours, mais aussi pour contribuer à la mise au point d'une politique de développement rural, en particulier dans la perspective d'une intégration de la Pologne à l'Union Européenne.

\section{BIBLIOGRAPHIE}

Dynamique sociale et mutations territoriales. Le cas de Mikołajki, 1991, LanguedocMazowsze, Université Montpellier III.

Lijewski T., Mikułowski B., Wyrzykowski J., 1998, Geografia turystyki Polski [La géographie du tourisme en Pologne], PWE, Warszawa.

Polska. Atlas agroturystyczny, 1999, Warszawa.

Rapport des enquêtes à Mikołajki, 1991, groupe Languedoc-Mazowsze, Warszawa (ronéo.) 